\title{
Production and modification of hadronic resonances measured with ALICE
}

\author{
Jihye Song (for the ALICE Collaboration)*i \\ Author affiliation: University of Houston \\ E-mail: Jihye.Song@cern.ch
}

\begin{abstract}
Measurements of the production of short-lived hadronic resonances are used to probe the properties of the late hadronic phase in ultra-relativistic heavy-ion collisions. Since these resonances have lifetimes comparable to that of the produced fireball, they are sensitive to the competing effects of particle re-scattering and regeneration in the hadronic gas, which modify the observed particle momentum distributions and yields after hadronisation. Having different masses, quantum numbers and quark content, hadronic resonances carry a wealth of information on different aspects of ion-ion collisions, including the processes that determine the shapes of particle momentum spectra, strangeness production, and the possible onset of collective effects in small systems. We present the most recent ALICE results on $\rho(770)^{0}, \mathrm{~K}^{*}(892), \phi(1020), \Sigma(1385)^{ \pm}, \Lambda(1520)$, $\Xi(1530)^{0}$ and $\Xi(1820)$ production at the LHC. They include measurements performed in pp, p-Pb and $\mathrm{Pb}-\mathrm{Pb}$ collisions at different energies, as well as the results from the LHC Run 2 with $\mathrm{Xe}-\mathrm{Xe}$ collisions at $\sqrt{s_{\mathrm{NN}}}=5.44 \mathrm{TeV}$. Collision energy and multiplicity differential measurements of transverse momentum spectra, integrated yields, mean transverse momenta and particle yield ratios are discussed in detail. A critical overview of these results will be given through comparisons to measurements from other experiments and theoretical models.
\end{abstract}

European Physical Society Conference on High Energy Physics - EPS-HEP2019 -

10-17 July, 2019

Ghent, Belgium
${ }^{*}$ Speaker.
${ }^{\dagger}$ ALICE Collaboration 


\section{Introduction}

Measurements of resonance particle production in relativistic heavy-ion collisions play an important role in understanding the properties of hadronic matter under extreme conditions. The short-lived hadronic resonances have a lifetime shorter or comparable to the lifetime of the fireball [1] and thus can undergo re-scattering and regeneration processes [2, 3], causing their yield to deviate from expected thermal model predictions [4]. Therefore, the study of resonances with different lifetimes is interesting to characterize the late stages of the fireball evolution. The ALICE detector is especially suited for the study of the Quark-Gluon Plasma (QGP) produced in relativistic heavy-ion collisions because of its exceptional particle identification capabilities. The study of strange resonant states, such as $\Xi(1530)$ and $\Xi(1820)$, can further reveal more exotic effects in heavy-ion collisions. The calculation from the FASTSUM Collaboration [5] shows potential parity doubling of strange resonant states, which could be a signature of chiral symmetry restoration in heavy-ion collisions.

\section{Analysis}

A complete and detailed description of the ALICE detector can be found in refs. [6, 7]. The main detectors that are relevant to this analysis cover the mid-pseudorapidity region $(|\eta|<0.9)$. They are the Time Projection Chamber (TPC), the Time-of-Flight detector (TOF), and the Inner Tracking System (ITS). Particle identification (PID) is performed by means of specific energy loss $(\mathrm{d} E / \mathrm{d} x)$ measurements in the gas filling the TPC and by means of time of flight measurements with the TOF detector. In addition, the V0 detectors (V0A covering $2.8<\eta<5.1$ and V0C covering $-3.7<\eta-1.7)$ consist of two sets of scintillators. They are used for triggering and measurement of multiplicity/centrality classes defined as percentiles of the distribution of the summed amplitudes in the $\mathrm{V} 0$ detectors $(\mathrm{V} 0 \mathrm{~A}+\mathrm{V} 0 \mathrm{C})[8]$.

The measurements of resonance production are performed at mid-rapidity $(|y|<0.5$ in pp, $\mathrm{Pb}-\mathrm{Pb}$ and $\mathrm{Xe}-\mathrm{Xe}$ collisions and $0<y_{c m}<0.5$ in $\mathrm{p}-\mathrm{Pb}$ collisions) as a function of the charged particle density, which is also measured at mid-rapidity for each multiplicity class. Invariant mass analyses are performed to measure resonances and Table 1 provides a list of resonances with their lifetimes, decay modes and branching ratios [9]. In the invariant-mass method one needs to estimate the combinatorial background, which is evaluated by using an event-mixing technique. After the background subtraction the raw yields are extracted from the signal distribution and then corrected for detector acceptance, tracking efficiency and branching ratio.

\begin{tabular}{cccccccc} 
& $\rho(770)^{0}$ & $\mathrm{~K}^{*}(892)^{0}$ & $\Sigma(1385)^{ \pm}$ & $\Xi(1820)^{\mp}$ & $\Lambda(1520)$ & $\Xi(1530)^{0}$ & $\phi(1020)$ \\
\hline Lifetime (fm/c) & 1.3 & 4.2 & 5.5 & 8.1 & 12.6 & 21.7 & 46.4 \\
Decay mode & $\pi^{+} \pi^{-}$ & $\mathrm{K}^{+} \pi^{-}$ & $\Lambda \pi^{ \pm}$ & $\Lambda \mathrm{K}^{-}$ & $p \mathrm{~K}^{-}$ & $\Xi^{-} \pi^{+}$ & $\mathrm{K}^{+} \mathrm{K}^{-}$ \\
B.R. (\%) & 100 & 66.6 & 87.0 & unknown & 22.5 & 66.7 & 49.2
\end{tabular}

Table 1: Lifetime values, reconstructed decay mode and branching ratio for measured resonance particles 


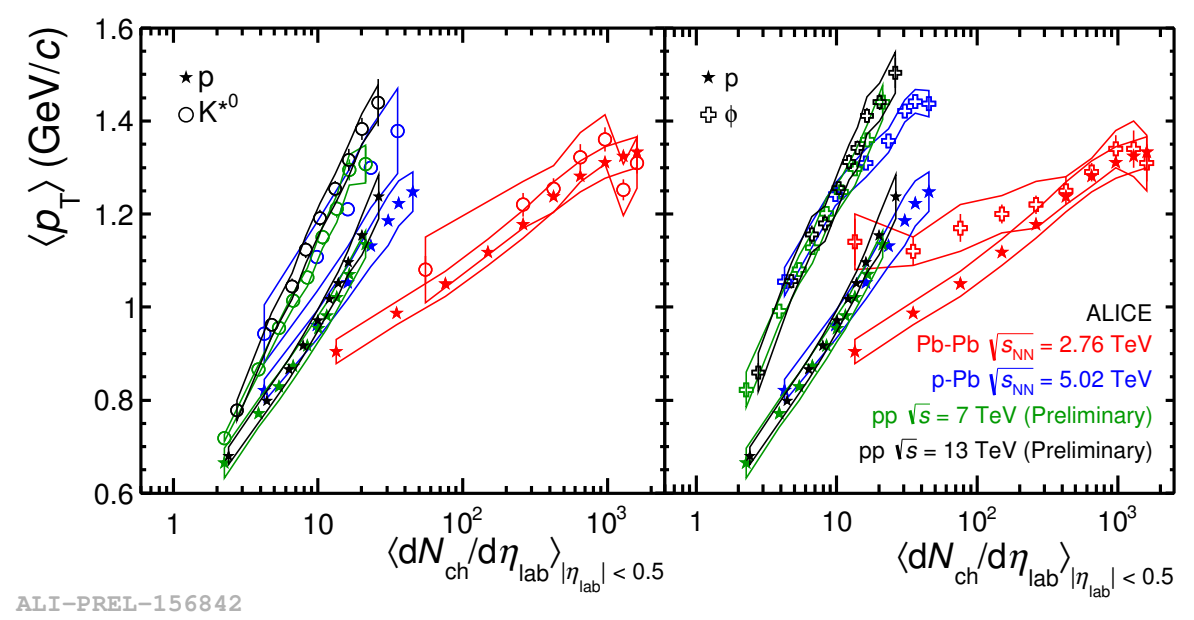

Figure 1: Mean transverse momentum $\left(\left\langle p_{\mathrm{T}}\right\rangle\right)$ of $\mathrm{K}^{* 0}, \phi$ and $\mathrm{p}$ in pp collisions at $\sqrt{s}=7$ and $13 \mathrm{TeV}, \mathrm{p}-\mathrm{Pb}$ at $\sqrt{s_{\mathrm{NN}}}=5.02 \mathrm{TeV}$ and $\mathrm{Pb}-\mathrm{Pb}$ at $\sqrt{s_{\mathrm{NN}}}=2.76 \mathrm{TeV}$ as function of multiplicity. The bars represent the statistical error and the lines represent the systematic error.

\section{Results and discussion}

The resonances are measured in several multiplicity classes in $\mathrm{pp}, \mathrm{p}-\mathrm{Pb}, \mathrm{Pb}-\mathrm{Pb}$ and $\mathrm{Xe}-\mathrm{Xe}$ collisions at different energies. The $p_{\mathrm{T}}$-integrated yields $(\mathrm{d} N / \mathrm{d} y)$ and mean transverse momenta $\left(\left\langle p_{\mathrm{T}}\right\rangle\right)$ for each multiplicity event class are obtained by integrating the $p_{\mathrm{T}}$-spectra to the measured range and by using a fit function to extrapolate the yields in the unmeasured $p_{\mathrm{T}}$ region. Figure 1 shows the $\left\langle p_{\mathrm{T}}\right\rangle$ of proton, $\mathrm{K}^{* 0}$ and $\phi$ as a function of the average charged particle multiplicity density $\left(\left\langle\mathrm{d} N_{\mathrm{ch}} / \mathrm{d} \eta\right\rangle\right)$ measured at mid-rapidity $(|y|<0.5)$ in pp collisions at $\sqrt{s}=7$ and $13 \mathrm{TeV}$ and compared with the results obtained in $\mathrm{p}-\mathrm{Pb}$ and $\mathrm{Pb}-\mathrm{Pb}$ collisions at $\sqrt{s_{\mathrm{NN}}}=5.02$ and $2.76 \mathrm{TeV}$, respectively. For the three particles, which have similar masses but different quark composition, an increase in $\left\langle p_{\mathrm{T}}\right\rangle$ from low to high multiplicity classes is observed. The same increasing trend of the $\left\langle p_{\mathrm{T}}\right\rangle$ as a function of the multiplicity is observed in pp collisions at $\sqrt{s}=7 \mathrm{TeV}$ and $13 \mathrm{TeV}$. A mass ordering of $\left\langle p_{\mathrm{T}}\right\rangle$ is found to be followed in central and semi-central $\mathrm{Pb}-\mathrm{Pb}$ collisions as expected from the hydrodynamic expansion of the system [10]. However, this breaks down for smaller systems. The increase in $\left\langle p_{\mathrm{T}}\right\rangle$ is steeper for smaller systems.

The yields normalized to the $\left\langle\mathrm{d} N_{\mathrm{ch}} / \mathrm{d} \eta\right\rangle$ of $\mathrm{K}^{* 0}$ and $\phi$ in pp collisions at $\sqrt{s}=7$ and $13 \mathrm{TeV}$, p-Pb collisions at $\sqrt{s_{\mathrm{NN}}}=5.02$ and $8.16 \mathrm{TeV}$ are shown as a function of multiplicity $\left(\left\langle\mathrm{d} N_{\mathrm{ch}} / \mathrm{d} \eta\right\rangle_{\left|\eta_{\text {lab }}\right|<0.5}\right)$ in Figure 2. The scaled integrated yield seems to be flat with multiplicity and independent of collision energy and system for $\mathrm{pp}$ and $\mathrm{p}-\mathrm{Pb}$ collisions within the uncertainties. This shows that the particle production is mainly driven by charged particle multiplicity irrespectively of collision system and energy.

The ratios of $p_{\mathrm{T}}$-integrated yields as a function of multiplicity are shown in Figure 3 for $\mathrm{pp}$, $\mathrm{p}-\mathrm{Pb}, \mathrm{Pb}-\mathrm{Pb}$ and Xe-Xe collisions, for $\rho^{0} / \pi, \mathrm{K}^{* 0} / \mathrm{K}, \Sigma^{* \pm} / \Lambda, \Lambda(1520) / \Lambda, \Xi^{* 0} / \Xi$ and $\phi / \mathrm{K}$. The yield ratios for short-lived resonances show a sizable dependence on the multiplicity. A clear suppression is observed for $\rho^{0} / \pi$ going from pp to central $\mathrm{Pb}-\mathrm{Pb}$ collisions at $\sqrt{s_{\mathrm{NN}}}=2.76 \mathrm{TeV}$ [11]. 

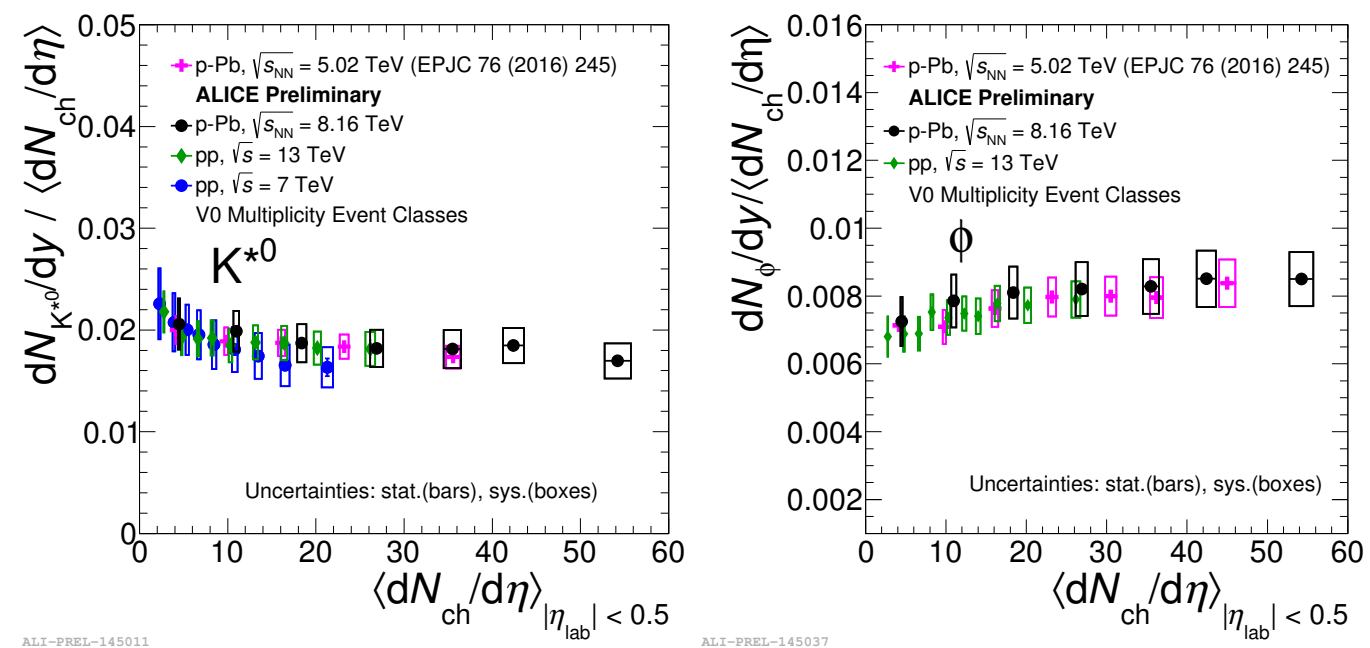

Figure 2: Integrated yields of $\mathrm{K}^{* 0}$ (left panel) and $\phi$ (right panel) normalized to $\left\langle\mathrm{d} N_{\mathrm{ch}} / \mathrm{d} \eta\right\rangle_{\left|\eta_{\text {lab }}\right|<0.5}$ in pp collisions at $\sqrt{s}=7$ and $13 \mathrm{TeV}$ and $\mathrm{p}-\mathrm{Pb}$ collisions at $\sqrt{s_{\mathrm{NN}}}=5.02$ and $8.16 \mathrm{TeV}$ for different multiplicity classes. The bars and the boxes represent the statistical and systematical error, respectively.

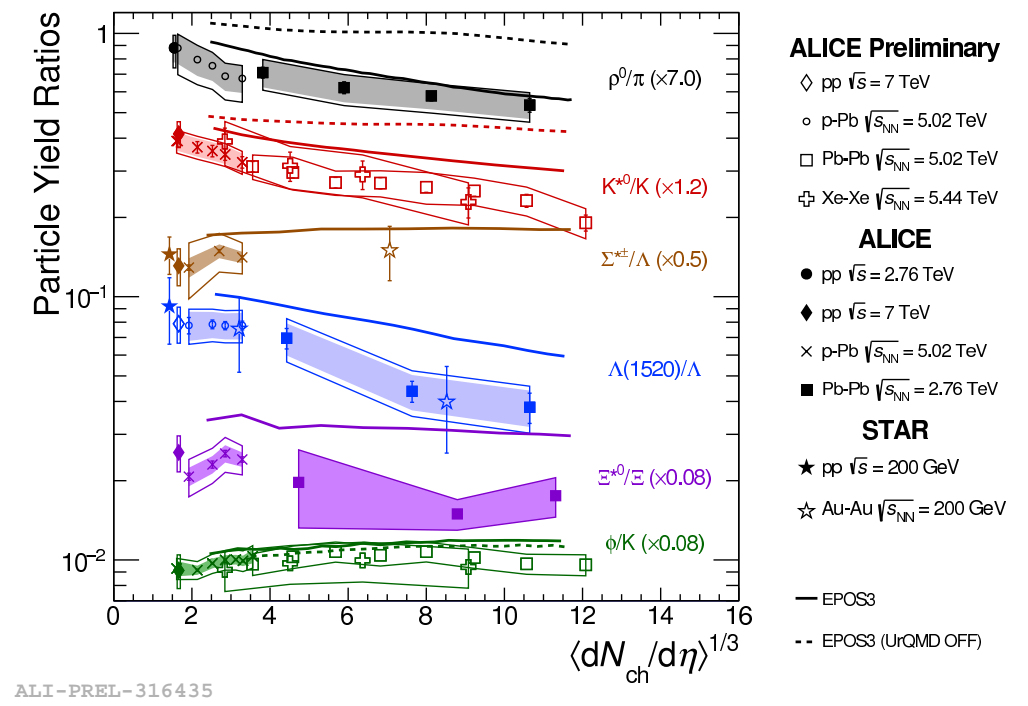

Figure 3: Ratio of resonance yields to those of long-lived hadrons is reported as a function of multiplicity for the hadronic resonances, $\rho^{0} / \pi[11], \mathrm{K}^{* 0} / \mathrm{K}, \Sigma^{* \pm} / \Lambda, \Lambda(1520) / \Lambda, \Xi^{* 0} / \Xi$ and $\phi / \mathrm{K}$ from inelastic pp, p-Pb, $\mathrm{Pb}-\mathrm{Pb}$ and $\mathrm{Xe}-\mathrm{Xe}$ collisions. The error bars show the statistical uncertainty, while the emtpy and darkshaded boxes show the total systematic uncertainty and the contribution uncorrelated across multiplicity bins, respectively. The measured data are compared to the ratios predicted by the EPOS3 model for $\mathrm{Pb}-\mathrm{Pb}$ collisions at $\sqrt{s_{\mathrm{NN}}}=2.76 \mathrm{TeV}$, where the late hadronic cascade is simulated with UrQMD. STAR data are also shown for the $\Sigma^{* \pm} / \Lambda$ and $\Lambda(1520) / \Lambda$ ratios. 
The EPOS3 event generator [12], which includes UrQMD [13] for the late-stage hadronic cascade is able to describe the evolution with multiplicity. The attempt to describe the $\rho^{0} / \pi$ ratio without UrQMD component fails to reproduce the data, with an overestimated and multiplicity-independent $\rho^{0} / \pi$ ratio [14]. The same behavior is observed in the $\mathrm{K}^{* 0} / \mathrm{K}$ ratio, where the suppression can be observed in a wider multiplicity range with data from pp at $\sqrt{s}=7 \mathrm{TeV}$ [15], $\mathrm{Pb}-\mathrm{Pb}$ at $\sqrt{s_{\mathrm{NN}}}=$ 5.02 TeV and Xe-Xe at $\sqrt{s_{\mathrm{NN}}}=5.44 \mathrm{TeV}$.

The ratios of $\Sigma^{* \pm} / \Lambda$ and $\Lambda(1520) / \Lambda$ show flat behavior in small systems from pp and $\mathrm{p}-\mathrm{Pb}$ collisions and the results are in agreement with previous measurements by STAR [17]. An energy dependence is not observed for these ratios from RHIC to LHC energies. Despite having a lifetime almost ten times longer than the $\rho^{0}$, the measurements of the $\Lambda(1520)$ production in $\mathrm{Pb}-\mathrm{Pb}$ reveal that it is suppressed with respect to the $\Lambda$ production [16], similarly to what is observed for other short-lived resonances. However, at present, EPOS3 predictions can only qualitatively describe the data, showing a similar decrease with multiplicity, but with a higher $\Lambda(1520)$ yield. Finally, there is no significant centrality dependence of the $\Xi^{* 0} / \Xi$ and $\phi / \mathrm{K}$ ratios with multiplicity for all measured systems. This is expected in the context of re-scattering, considering that the $\Xi^{* 0}$ baryon and the $\phi$ meson live longer than the expected fireball lifetime and therefore their decay daughters will not undergo re-scattering.
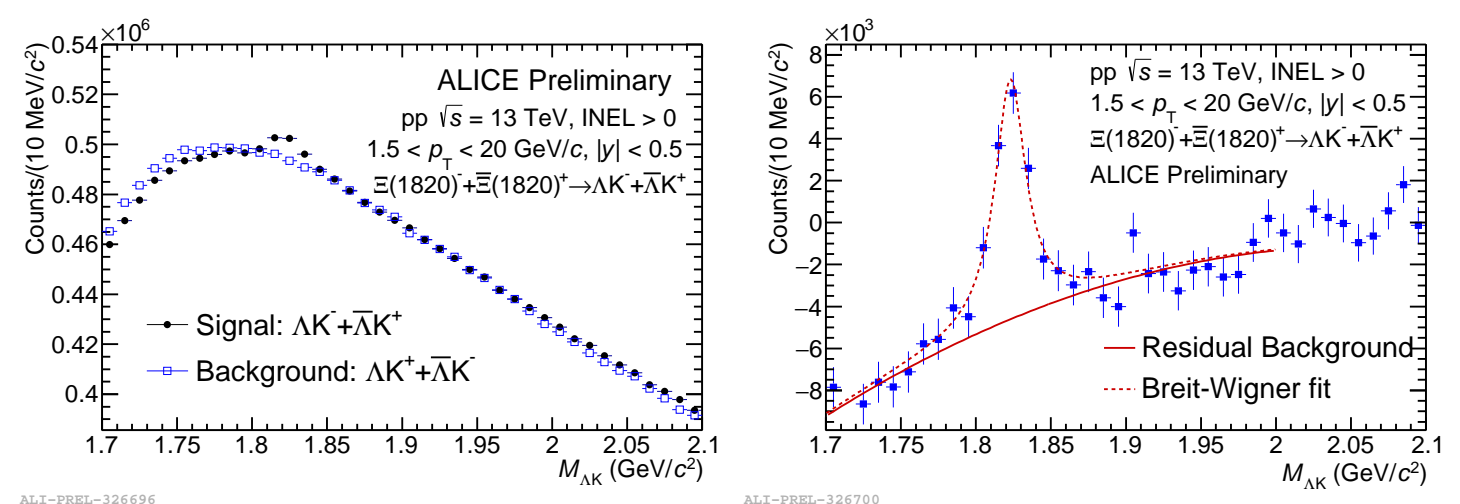

Figure 4: (Left) The $\Lambda K$ Invariant mass distribution for $1.5<p_{\mathrm{T}}<20 \mathrm{GeV} / \mathrm{c}$ in pp collisions at $\sqrt{s}=13 \mathrm{TeV}$. (Right) The invariant mass distribution after subtraction of the background. The dashed curve represents the combined fit, while the solid line describes the residual background.

The first measurement of $\Xi(1820)$ from collider experiments is shown in Figure 4. Calculations from FASTSUM Collaboration [5] suggest a shift in the mass of the $\Xi(1820)$, which could be a signature of chiral symmetry restoration in heavy-ion collisions. The masses of positive-parity states are largely temperature-independent while the masses of negative-parity states drop and become near-degenerate with the corresponding positive-parity mass close to the critical temperature. Because the $\Xi(1820)$ has negative parity, the observation of a mass shift, width broadening, or a change in its yield relative to the positive-parity $\Xi(1530)$ can be a key measurement to study chiral symmetry restoration. 


\section{Summary}

The ALICE collaboration reported results on resonance production as a function of multiplicity. The measurement of hadronic resonances is also performed for various systems (from pp, $\mathrm{p}-\mathrm{Pb}, \mathrm{Pb}-\mathrm{Pb}$ and $\mathrm{Xe}-\mathrm{Xe})$. The increase of $\left\langle p_{\mathrm{T}}\right\rangle$ is observed from low to high multiplicity classes and a mass ordering of $\left\langle p_{\mathrm{T}}\right\rangle$ is found in central $\mathrm{Pb}-\mathrm{Pb}$ collisions. The $p_{\mathrm{T}}$-integrated yields of $\mathrm{K}^{* 0}$ and $\phi$ normalized to the average charged particle multiplicity appear to be constant as a function of the charged particle multiplicity in $\mathrm{pp}$ and $\mathrm{p}-\mathrm{Pb}$ collisions. The ratios of $p_{\mathrm{T}}$-integrated particle yields for various resonances are measured and the $\rho^{0} / \pi, \mathrm{K}^{* 0} / \mathrm{K}$ and $\Lambda(1520) / \Lambda$ ratios exhibit a decrease from $\mathrm{pp}$ and peripheral $\mathrm{Pb}-\mathrm{Pb}$ to central $\mathrm{Pb}-\mathrm{Pb}$ collisions. This behavior can be explained by the dominance of re-scattering of decay daughters over regeneration in the hadronic phase. A comparison of the resonance suppression with an EPOS3 prediction including UrQMD indicates that re-scattering and regeneration processes are required to describe the measurements. The first measurement of $\Xi(1820)$ is presented and will be compared to the corresponding positive-parity particle to study chiral symmetry restoration.

\section{References}

[1] C. Markert, R. Bellwied, and I. Vitev Phys. Lett. B669 (2008) 92-97

[2] M. Bleicher and J. Aichelin Phys. Lett. $B 530$ (2002) 81-87

[3] M. Bleicher and H. Stoecker J. Phys. G30 (2004) S111-S118

[4] J. Stachel, A. Andronic, P. Braun-Munzinger, and K. Redlich J. Phys. Conf. Ser. 509 (2014) 012019

[5] G. Aarts, C. Allton, D. Boni, S. Hands, B. Jäger, C. Praki, and J.I. Skullerud. EPJ Web Conf. 171 (2018) 14005

[6] K. Aamodt et al. (ALICE Collaboration), JINST 3 (2008) S08002

[7] B. Abelev et al. (ALICE Collaboration), Int. J. Mod. Phys. A29 (2014) 1430044

[8] B. Abelev et al. (ALICE Collaboration), Phys. Rev. C88 (2013) 044909

[9] M. Tanabashi et al. (Particle Data Group), Phys. Rev. D 98, 030001 (2018)

[10] C. Shen, U. Heinz, P. Houvinen, and H. Song Phys. Rev.C84 (2011) 044903

[11] S. Acharya et al. (ALICE Collaboration), Phys. Rev. C99 064901

[12] K. Werner, B. Guiot, I. Karpenko, and T. Pierog Phys. Rev. C89 (2016) 064903

[13] M. Bleicher et al. J. Phys. G25 (1999) 1859-1869

[14] A. G. Knospe, C. Markert, K. Werner, J. Steinheimer, and M. Bleicher Phys. Rev. C93 (2016) 014911

[15] B. Abelev et al. (ALICE Collaboration), Eur. Phys. J. C72 (024905)2012) 2183

[16] S. Acharya et al. (ALICE Collaboration), Phys. Rev. C99 024905

[17] B. I. Abelev et al. (STAR Collaboration), Phys. Rev. Lett. 97 (2006) 132301 